\title{
Effect of Exercise Type on Vascular Stabilization Factor in Ischemic Stroke Patients
}

\author{
Mahshid Kadkhodaei Khalafi ${ }^{1}$, Nader Shakeri², Maziar Shojaee³, Farshad Ghazalian ${ }^{4}$ \\ ${ }^{1}$ Department of Sport Physiology, Faculty of Humanities and Social Sciences, Islamic Azad University, \\ Sciences and Research Branch, Tehran, Iran. ${ }^{2}$ Department of Sports Physiology, Faculty of Physical \\ Education and Sport Sciences, Humanities Building, Islamic Azad University, Science and Research Branch \\ of Tehran, Iran. ${ }^{3}$ Department of Internal Neurology, Shahid Beheshti University of Medical Sciences, Tehran, \\ Iran. ${ }^{4}$ Department of Sports Physiology, Faculty of Physical Education and Sport Sciences, Humanities \\ Building, Islamic Azad University, Science and Research Branch, Tehran, Iran.
}

\section{ABSTRACT}

\section{BACKGROUND}

Stroke is one of the leading causes of mortality and disability in the world. The angiogenesis strategy is a new therapeutic approach to exercise in these patients. The purpose of this study was to investigate the effect of continuous exercise on serum levels of vascular stabilizing factor and its consequences.

\section{METHODS}

In this randomized clinical trial study, 30 patients with stroke who volunteered to participate in the study, aged 45 - 65 years, were selected by convenience sampling and non-random sampling method. Subjects were randomly selected. The patients were randomly divided into intervention group ( 4 weeks of continuous exercise in $40-60$ minutes pedal with $60-65 \% \mathrm{VO}_{2}$ peak intensity for 5 days per week and physiotherapy) and control group (physiotherapy only). Blood angiogenesis and motor function levels were measured at baseline and twenty-eight days after the last exercise session. Data was analyzed by using Stata software version 13 at the significance level of 0.05 .

\section{RESULTS}

In this trial, thirty patients completed the study period, and were included in the analysis. The mean ages of patients in the control and intervention groups were 55.66 (S.D.: 6.69) and 61.46 (S.D.: 6.47) years, respectively. Serum levels of angiopoietin-1 increased after exercise at alpha level $(\mathrm{P}=0.011)$. The reduction of the Modified Rankin Scale was obtained as a secondary consequence $(\mathrm{P}=0.001)$.

\section{CONCLUSIONS}

Continuous exercise training over four weeks can improve the prognosis of patients by altering the levels of angiogenesis-stabilizing factor in decreasing the symptoms of stroke.

\section{KEY WORDS}

Stroke, Rankin Scale, Continuous Exercise, Angiopoietin-1
Corresponding Author:

Nader Shakeri,

Assistant Professor

Department of Sport Physiology,

Faculty of Humanities and Social Sciences, Islamic Azad University, Sciences and

Research Branch, Tehran, Iran.

E-mail: nsprofsport@gmail.com

DOI: $10.14260 / \mathrm{jemds} / 2020 / 509$

How to Cite This Article:

Khalafi MK, Shakeri $N$, Shojaee $M$, et al. Effect of exercise type on vascular stabilization factor in ischemic stroke patients. J Evolution Med Dent Sci 2020;9(33):2345-2351, $10.14260 /$ jemds/2020/509

Submission 28-03-2020,

Peer Review 07-07-2020,

Acceptance 13-07-2020,

Published 17-08-2020.

Copyright (c) 2020 JEMDS. This is an open access article distributed under Creative Commons Attribution License [Attribution 4.0 International (CC BY 4.0)] 


\section{BACKGROUND}

Stroke is known to be the second leading cause of mortality as well as an important cause of disability that imposes huge economic costs on the society.(1,2) In 2016, 43.17\% of stroke cases were ischemic and $10.50 \%$ of them were haemorrhagic.(2) Quality of life is of high significance as an important index for evaluating individual health.(3) From among pro-angiogenic growth factors, Angiopoetin-1 (ANGPT1 or Ang 1) is unique; it is capable of rebuilding the arteries through an extreme organization of angiogenesis and tightening of endothelial junctions by activating both vascular Tie2 and non-muscular signaling integrilin, and it is also considered an acceptable choice for vascularization of nerves and artery protection. (4) Moreover, it is required for the proper organization of the maturity of newly formed vessels. (5) Through the angiogenesis process, the vessels are reproduced, and at the occurrence of ischemia, the newly-formed vessels join each other in a primary vascular network as a capillary network.(6)

The newly formed vessels are also capable of reducing brain edema. Despite the existence of pharmaceutical and chemical treatments as well as surgery, the rehabilitation of patients suffering from stroke is of great significance for both medical groups and non-medical groups (physiologists, sports coaches. Despite the progresses made for understanding cellular molecular mechanisms and pathophysiology of stroke in the last decades, only $4 \%$ of the patients are capable of receiving the pharmaceutical treatment of thrombolytic therapy for acute ischemic strokes.(7) Physical exercise can directly bring about some intracellular incidents,(8) through two mechanisms i.e. increasing tensile stress on the brain vessel walls and activating the related signals and increasing the metabolic activity of brain nerve cells. The interaction of these two mechanisms will simultaneously result in increasing endothelial growth factors and intracellular waterfall factors in mitochondrion, elevating the antioxidant activity, and changing HSPs levels (Heat Shock Protein) with (BDNF) Brainderived Neurotrophic Factor. Thus, the blood flow increases, and through increasing the complexity of brain vessels and changing the neuronal volume, the angiogenesis process results in increased brain health and nerve cells in the form of improved learning, memory, and performance.(9)

The main focus of the exercises is on weight bearing and balance and performance methods. In the chronic stage with acute stroke, the researchers are being conducted on animal samples. Moreover, in the acute stage of stroke, few studies have been conducted.(10,11) The researchers have investigated the role of early initiation of exercises on patients suffering from acute stroke from the admission time with Modified Rankin Scale (MRS); they have observed that the experimental group (with the early initiation of exercises), in comparison to the control group, provided better results after three months of exercise.(12) Matsuda et al have stated that in brain-induced injury cases, continuous exercise on treadmill for 28 days brings about changes in the motor behaviour and function in mice (in less than 24 hours) by changing the cellular expression levels of anti-platelet endothelial cells' adherent molecule, neuron growth factor, and Caspase 3; this reduces the nerve defect and infarct volume and may result in the improvement of the central nervous system.(13) On the other hand, another study has been conducted by Seok Woo that had different results. Seok Woo's study focused on muscular tonicity balance and static balance in 22 chronic stroke patients. The patients walked on a treadmill in water and their results were compared with those of the patients walking on treadmill being on the ground. The exercise protocol was 30 minutes for four weeks and five times a week; the researcher did not observe a significant difference in terms of muscular power between healthy individual and patients suffering from muscular paresis.(14) Given the existing different results and since $96 \%$ of the patients do not have access to a pharmaceutical treatment, it is essential to provide substitute therapies including sports therapy.

The present study aims at investigating the effect of exercise type on the serum level of vascular stabilization factor in less than 6 hours and before the initiation of cell death and progress of infarct volume by applying the investigation of changes arising from stroke with MRS.

\section{METHODS}

\section{Study Design}

The present study is parallel groups randomized clinical controlled trial study. We followed the CONSORT checklist to report the results of this trial. This study was approved by the Ethical Committee of Shaheed Beheshti University of Medical Sciences with code: IR.SBMU.RETECH.REC.1397.1018 and also it was registered in Iranian Registry of Clinical Trials with code: IRCT20100518003943N1.

\section{Eligibility Criteria and Participants}

From among the patients diagnosed with stroke, the samples were selected with convenience sampling method. The patients were selected from those who had referred to the emergency unit of Loghman-e Hakim Hospital, Tehran, Iran, from October 2018 to February 2019. Having acquired the inclusion criteria of the present study, the participants were selected (in less than 24 hours after being admitted) with the diagnosis of ischemic stroke in the middle cerebral artery (based on brain CT Scan) by a neurologist and having a history of at least one atherosclerosis cause. The samples were selected after expressing their willingness and motivation for participating in a sports program. Study participants were randomly divided into control and intervention groups.The samples' level of consciousness was required to be 12-15 based on Glasgow Coma Scale.

The patients with a history of drug addiction, coagulation disorders, cancer, end stage renal disease, liver problems, heart and breathing problems, a fever of higher than 38 degrees, skeletal anomalies, vascular aneurysm, previous strokes, open wound and brain injuries, low level of consciousness, mental retardation (based on the medical records) were excluded from the study. Moreover, the researchers excluded the patients who were present in the initial blood sampling, but withdrew from the study for various reasons including being discharged on one's will, refusing to proceed the therapy, initiating non-standard 
therapies like applying leech, taking supplements that change blood concentration, losing motivation and life expectancy, withdrawing from the sports protocol, and the incidence of a new stroke.

\section{Interventions}

After becoming familiar with the exercise environment and doing warm-up for two minutes, the participants of the continuous exercise group conducted continuous aerobic exercise for 40-60 minutes on a rehabilitation bike with the severity of 60-65 percent of the maximum submaximal consumed oxygen for five days a week. Moreover, no overload was done; the workload was 2250 kilo-joules based on the exercise protocol offered by Martin Gibala in 2012.(15) After the ride, the participants conducted the cooling process for 2 minutes. The exercise was done for 28 days.

The control group consisted of 15 participants, and they had no exercise. The patients were provided with stationary rehabilitation foot bikes belonged to Turbo Fitness Company (Germany) and Keep Fit common bikes (China); both types had LCD monitors. For the prevention of possible falls, the patients were able to sit on the seat and hold the handles; they could also be supported through attaching wrist straps and leaning on a backrest. The bikes have remote controls not accessed by the participants, so that the patients cannot voluntarily switch off the machine. However, the exercise ends when there is a hemodynamic change, or the participant announces tiredness. Both control and intervention group were undergoing physiotherapy and received the same drug and diet protocol of stroke prescribed by their specialists.

\section{Outcomes and Measurements}

The participants filled out an informed consent, a general characteristics form, and a sports questionnaire. Moreover, the researchers investigated the participants' health and disability arising from the brain injury with MRS. MRS is a commonly used scale for measuring the degree of disability or dependence in the daily activities of people who have suffered a stroke. The MRS scale runs from 0 to 6 and ranging from the lack of symptoms of disability to severe disability and death.(16)

All samples were under Vectra cardiac and respiratory monitoring system equipped with pulse oximetry for the serial control of blood pressure, pulse, and blood oxygen percentage. Since the second phase of saving nerved cells and reestablishing the blood flow arising from ischemia starts 6 to 72 hours later, interventions toward reversing the ischemic process is of high significance in this time span.(2) As much as 5 cc blood was taken from the patients through their antecubital vein of right hand (at a sitting position) after being admitted and the initiation of monitoring in the emergency unit as well as on the $28^{\text {th }}$ day at the clinic.

After centrifuging the blood, the patients' serum was frozen at the temperature of -70 degrees, and the serum level of angiopoietin-1 was measured with ELISA method and Zellbio Kit (with the range of 31.35-2000 picogram/millilitre and sensitivity of 1.03 picogram/millilitre) at Endocrinology and Metabolism Research Institute in Tehran. All the stages of the study were conducted in the Occupational Therapy Clinic of Loghman-e Hakim Hospital (Tehran).
For reducing the errors and having a more accurate supervision, the exercises were conducted in the presence of the specialist and researchers from 3 to 7 p.m. at the temperature of 25 degrees and humidity of 35-42 degrees and the atmospheric pressure of 700 to 1060 Pascal (based on the weather report of Tehran). The vital signs, underlying signs, and the disability extent of stroke patients were measured before the initial six hours and at the end of $28^{\text {th }}$ day by MRS. Lack of change in terms of the individuals' motor function and their weakening were considered as bad prognosis, and improved motor function was regarded as good prognosis.

\section{Randomization}

By an online application called "Sealed Envelope", the participants were randomly allocated into two groups by permuted block randomization which guarantee the allocation concealment.

\section{Statistical Procedure}

Initially, the Shapiro-Wilk test was used to test the normality assumption of the data. Due to the normality violation in angiopoietin-1, nonparametric tests (such as Mann-Whitney U and Wilcoxon signed-rank) were used to compare the differences among two groups as well as before and after intervention. Because of normal distribution, and the mean of MRS were compared with two independent t-test (comparison of two groups) and paired t-test (comparison of before-after intervention). The analysis was performed by Stata 13 (Stata Corp. LLC., College Station, TX, USA). The significance level was considered to be less than 0.05 .

\section{Sample Size}

In this pilot study, we expected that as a minimum a total of 30 cases are needed (15 in each group) to achieve a 1000 units difference in average of the expression of angiopoietin-1 level between the two groups and the standard deviation of 800. A power of $80 \%$ and a $\mathrm{P}<0.05$ level of significance were considered for this study. The sample size was calculated with Stata software version 13 as below:

\section{Performing Iteration}

Estimated sample sizes for a two-sample means test $t$ test assuming sd $1=\mathrm{sd} 2=\mathrm{sd}$

Ho: $m 2=m 1$ versus Ha: $m 2 !=m 1$

\author{
Study Parameters \\ Alpha $=0.0500$ \\ Power $=0.8000$ \\ Delta $=3.0619$ \\ $\mathrm{m} 1=4200.0000$ \\ $\mathrm{m} 2=5200.0000$ \\ $\mathrm{sd}=800.0000$
}

\section{Estimated Sample Sizes}

$\mathrm{N}=24$

$\mathrm{N}$ per Group $=12$ 


\section{RESULTS}

In this randomized clinical trial, thirty patients were included the study and all of them completed the study period and included in the analysis. At first, in Table 1, the demographic and baseline characteristics of participants were provided for each group. Bases on Table 1, the mean age of patients in control and intervention groups were 55.66 (S.D.: 6.69) and 61.46 (S.D.: 6.47) years, respectively. Other baseline characteristics were presented in Table 1.

\begin{tabular}{|c|c|c|}
\hline Variables & $\begin{array}{c}\text { Control Group } \\
(\mathbf{N = 1 5 )}\end{array}$ & $\begin{array}{c}\text { Intervention } \\
\text { Group (N=15) }\end{array}$ \\
\hline Age (years) & $61.46 \pm 6.47$ & $55.66 \pm 6.69$ \\
Body Mass Index $\left(\mathrm{Kg} / \mathrm{m}^{2}\right)$ & $25.06 \pm 2.64$ & $28.53 \pm 2.79$ \\
Height $(\mathrm{cm})$ & $167.84 \pm 5.26$ & $168.60 \pm 3.13$ \\
Systolic Blood Pressure (mm Hg) & $159.00 \pm 19.00$ & $153.00 \pm 21.00$ \\
Diastolic Blood Pressure (mm Hg) & $91.00 \pm 12.00$ & $84.00 \pm 8.00$ \\
Heart rate (in minutes) & $96.00 \pm 9.00$ & $85.00 \pm 7.00$ \\
Breathing rate (per minutes) & $18.00 \pm 2.00$ & $16.00 \pm 2.00$ \\
\hline Table 1. Baseline Characteristics of the Participants \\
\hline The values are mean and standard deviation \\
\hline
\end{tabular}

The means of MRS were compared in Table 2. As it was shown in Table 2, after the intervention compared to the baseline, a significant decrease was observed in both control $(p=0.028)$ and intervention groups $(p=0.001)$. Also, a significant difference was observed between the two groups after the intervention in terms of MRS, so that the mean of MRS in the intervention group was significantly lower than the control group $(\mathrm{P}=0.002$, Figure 1$)$.

\begin{tabular}{|cccc|}
\hline Groups & MRS at Baseline & MRS at the End of Study & P Value \\
Control group & $3.2(0.56)$ & $2.8(0.77)$ & 0.028 \\
Intervention group $\quad 2.7(0.72)$ & $2.0(0.53)$ & 0.001 \\
Two independent t test p value $=0.002$ & & \\
The values are mean and standard deviation \\
\hline \multicolumn{3}{|r|}{ Table 2. Modified Rankin Scale (MRS) in the Groups both } \\
before and after the Intervention
\end{tabular}

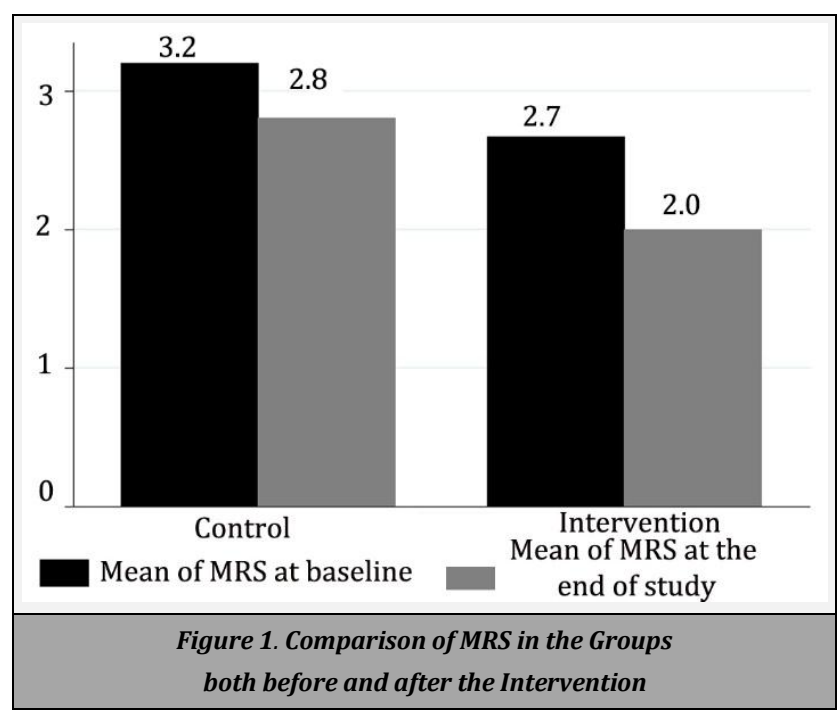

Since the results of the Shapiro Wilk test showed that the distribution of angiopoietin-1 level was not normal, Wilcoxon signed-rank test was used to compare before and after in each group (paired comparison) and Mann-Whitney U test was used to compare between the two groups. In the intervention group, Wilcoxon signed-rank test indicated that the angiopoietin-1 level was significantly increased at the end of intervention rather than baseline $(\mathrm{P}=0.011$, Table 3$)$, and also, in the control group, the angiopoietin-1 level at the end of study reduced in comparison to that of the baseline $(\mathrm{P}=0.002$, Table 3). Moreover, the results of Mann-Whitney $U$ test indicated that there is a significant difference in terms of angiopoietin-1 level among two groups at the end of intervention, so that angiopoietin-1 level at the end of study, in the intervention group was significantly higher than that of the control group $(\mathrm{P}=0.372$, Table 3, Figure 2$)$.

\begin{tabular}{|cccc|}
\hline Groups & $\begin{array}{c}\text { Angiopoietin-1 } \\
\text { at Baseline }\end{array}$ & $\begin{array}{c}\text { Angiopoietin-1 at } \\
\text { the End of Study }\end{array}$ & P Value \\
\hline Control group & $4456(3869)$ & $4184(3480)$ & 0.002 \\
Intervention group & $4504(3243)$ & $5119(2596)$ & 0.011 \\
\hline Mann-Whitney U test p value $=0.372$ \\
\hline \multicolumn{2}{|c|}{ The values are median and Inter quartile range (IQR) } \\
\hline \multicolumn{3}{|c|}{ Table 3. Comparison of Angiopoietin-1 Level in the Groups } \\
both before and after the Intervention \\
\hline
\end{tabular}

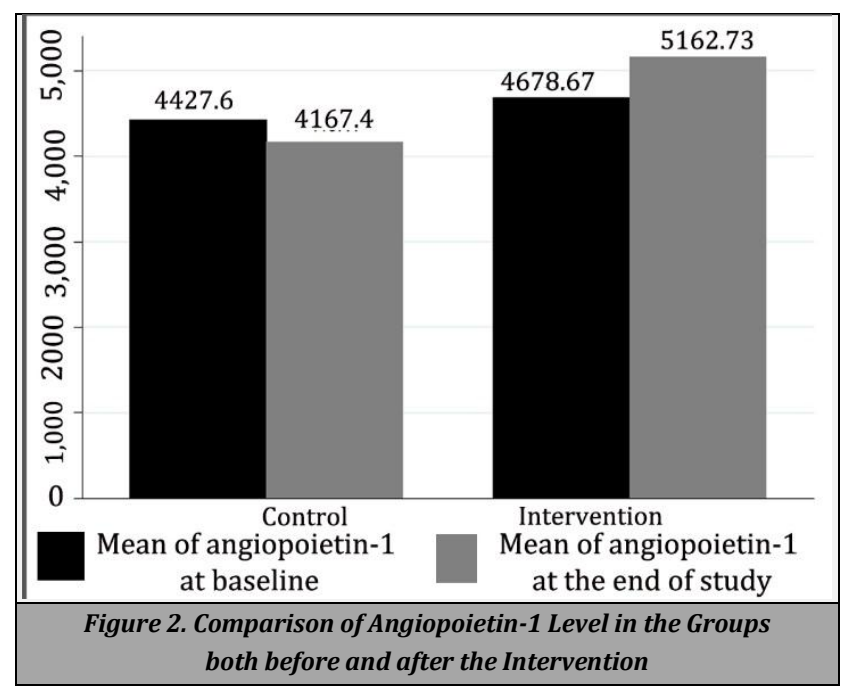

\section{DISCUSSION}

The complicated pathophysiology of stroke is in three phases (i.e. blood flow disorder, infarction progress, and chronic stage) and involves toxicity oxidation mechanisms, inflammatory channels, and ionic imbalance in brain nerve cells. The integrity of the blood-brain barrier has an important role in the structural resistance and maintaining the proper permeability of cerebral veins.(17) The significant feature of the second phase of stroke after 72 hours is the development of infarction core from the center to penumbra. The mechanisms of infarction progress include the toxicity arising from high cellular excitability, inflammation, increased production of free radicals, mitochondrial dysfunction, and oxidative stress. At the incidence of ischemia, hypoxia, and ATP shortage, the sodium potassium pump and calcium pump will suffer from dysfunction. Thus, excessive sodium and calcium ions intake from calcium channels will make the cells extremely excitable; this results in excessive release of glutamate to the synaptic space. Due to the lack of energy, the cells are capable of glutamate uptake from the synapses. When glutamate remains in the extracellular parts and calcium accumulates widely in this area, one can conclude that there will be deadly intracellular disorder and cellular death.(18) The formation and production of effective factors involved in supplying vascular reperfusion can occur by cellular stimulation and organization in muscles and brain. In the formation and reproduction 
process, small secondary vessels join each other as a capillary network.(6,7) In the vascular angiogenesis, Angiopoietin-1 is directly connected to non-vascular integrin and extra-cellular matrix by increasing tyrosine kinase-2. Moreover, Angiopoietin-1 will interact with the endothelial factor of cellinduced vascular growth, and it will cause a tight connection of pericytes without vascular leak to ischemic issues.(4) In the review article conducted by Mark \& Austin, it has been reported that doing sports and exercises has a significant role in reducing the inflammatory reaction and cytokines that prevent brain injury and oedema and protect brain against excitable factors and intensification of rapid reperfusion of vessels in samples with ischemic stroke on parameters of brain lesion volume.(19) In another study, Yong et al (2014) have observed that exercise reduces neuronal disorders and infarction volume in rats.(20) In a study conducted by Ding et al (2004), it was observed that exercise neuroprotection and neural resistance against the stroke-induced brain injury.(21) Moreover, by increasing the expression of collagen IV, exercise results in the improvement of basal lamina- 9 in the bloodbrain barrier (2008).(22) The early-initiated physical rehabilitation in the animal sample with acute stroke has been investigated by Zhang et al; this paved the way for the clinical implementation in human samples.(23)

After conducting a systematic review, it was concluded that the existing gap of the present knowledge derived from the investigated studies lies in the management of physical exercise and exercise prescription in stroke survivors by focusing on aerobic exercises in 24-72 hours after the stroke diagnosis. The present study aims at investigating the effect of early-initiated exercise in the acute stage of stroke and before the incidence of the second phase of infarction progress in less than 6 hours after the obstruction on the serum level of one of the angiogenesis indices and its consequences. After implementing the aerobic endurance exercises for 4 consecutive weeks, the serum level of Angiopoietin-1 increased significantly in comparison to the serum level measured at the admission of the patient. In line with the present study, the same protocol was conducted for 4 weeks with Wingate bikes; it led to increased oxidative capacity of skeletal muscle, tissue glycogen storage, and thus improved performance results and increased time of six-spot step test.(16) Moreover, it seems that aerobic exercises increase the ions of the calcium combined with nitric oxide in the muscle tissue.(23) It is worth noting that these patients underwent the same pharmaceutical treatment. It is obvious that anticoagulant drugs affect blood dilution, and calcium blockers influence calcium storage process in the contraction of smooth muscles of blood vessel wall. Yun-Hong Ding et al conducted a trial on Sprague Dawley rats with the exercise protocol of 30 minutes a day on a treadmill for one week, three weeks, and six weeks. They observed a significant increase in mRNA expression of growth factor and Angiopoietin 1 and 2 for one week, and neural defect and cerebral infarct volume reduced at a remarkable extent.(21)

The findings obtained from the study conducted by Nourshahi et al indicate that exercise prevents ischemia in brain and reduced injury extent is likely due to post-exercise angiogenic factors.(24) One of the main deterrents of brainspecific angiogenesis is the protein group coupled with protein $G$ that bond with its own Ligand at the outer surface of the cells, and Adrenalin, Adenosine, and opium transform and become activated. This protein is involved in the nervous messaging path and CAMP.(25) As the stimulator of endorphin and adrenaline secretion, exercise can change the signalling route of dihydropyrimidine receptors given the production of nitric oxide arising from hypoxia; exercise will result in increased secretion of endothelial growth factor. Thus, it can be claimed that a four-week aerobic endurance exercise affects brain reperfusion. The secretion of vascular endothelial growth factor is highly affected by tissue hypoxia and normoxia.(23) By applying distance shift, Vogel et al provided a new model by suggesting two methods of optional and compulsory treadmill running in rats for four weeks.(26) Nox4 maintains the vascular endothelial growth factor expression and prevents the increased expression of Angiopoietin-1.(26) In the sports studies, it has been indicated that a main part of higher endothelial factor content was in hypoxia conditions.(27) It is likely that in this study, effective factors such as low number of samples, exercise hardship, kinds of exercise, and injured brain area have been effective in the results obtained. The rehabilitation program results in an improved quality of life in physical performance, public health, and psychological and social performance. Moreover, thanks to the individuals' quick return to the society, physical exercise is greatly helpful in reducing the costs. $(3,8)$ Preconditioning with exercise on treadmill was conducted in the animal model suffering from induced stroke in middle cerebral artery for five days a week for 40 minutes a day and the speed of 18 meters a minute for five weeks; the investigation of infarct volume, neurological disorders, and sensory-motor disorders indicated that exercise functions like a neuroprotector in animals.(28) It is obvious that the cold temperature of the environment affects the individuals' inactivity and intensification of factors causing vascular accidents; this is in line with the findings of similar studies.(29)

Physical exercise is likely to reduce the complications arising from vascular injuries by increasing the blood flow in muscles, stimulating the receptors in muscles, and secreting hormones stimulating adrenaline, and stimulating calcium receptors at the cellular level. Moreover, it can be concluded that the duration of exercise is likely to affect the release and maintaining of serum volume in progenitor cells. However, one cannot exclusively attribute the reduction of the symptoms observed to the increased volume of Angiopoietin1. As it has been stated, the extent of brain's injured areas and the hardship and type of exercise affect the release of factors and reversing the tissue perfusion with new angiogenesis, and they can be different for different age ranges; this calls for conducting further studies.

\section{Research Limitations}

The present study suffered from a serious limitation; it was impossibile to directly obeserve the brain vascular changes and conduct a cellular signalling investigation in the human sample.

\section{Suggestions}

It is recommended that exercise interventions be conducted for more than 4 weeks. Moreover, the researcher suggests that effectiveness of exercise be conducted through measuring the 
infarct level with laser flow meter and providing an image of brain vessels or angiography CT.

\section{CONCLUSIONS}

Continuous exercise training for four weeks can improve the general quality of life in stroke patients by reducing MRS and increasing the stabilization factor of Angiopoietin-1 and the related domains. Moreover, it can lead to better understanding of reduction of brain injury arising from vascular ischemia.

The present research project is a clinical trial extracted from the doctoral dissertation in sports physiology. The researchers would like to express their sincere gratitude to the professors, patients, laboratory personnel, neurology staff, and clinical development research staff of Loghman Hakim Hospital for their expertise and assistance.

The Ethics Committee of Shahid Beheshti University of Medical Sciences and Health Services approved the study; IR. SBMU. RETECH. REC.1397.1018. IRCT code: 20100518003943N1.

\section{REFERENCES}

[1] Talebi SHA, Nabipour I, Vafaju F, et al. Hyperlipidaemic disorders and cerebrovascular accidents in the province of bushehr: a prospective study. Journal of Diabetes and Metabolic Disorders 2002-2003;2(1):31-8.

[2] Kasper DL, Fauci AS, Hauser SL, et al. Harrisons Manual of Medicine. 19th edn. McGraw-Hill Education 2016.

[3] Ganji MK, Hosseini E, Nasiri AH, et al. The effect of rehabilitation on the quality of life of patients with stroke in faculty of rehabilitation. Isfahan University of Medical Sciences, Isfahan, Iran. 2016.

[4] Koh GY. Orchestral actions of angiopoietin-1 in vascular regeneration. Trends in Molecular Medicine 2013;19(1):31-9.

[5] Ramezani T, Baharara J. A review on angiogenesis in tumor. J Cell and Tissue 2014;5(1):89-100.

[6] Dvorak HF. Angiogenesis. Journal of Thrombosis and Haemostasis 2005;3(8):1835-42.

[7] Fonarow GC, Smith EE, Saver JL, et al. Timeliness of tissue type plasminogen activator therapy in acute ischemic stroke. Circulation 2011;123:(7):750-8.

[8] Feng R, Zhang $M$, Wang $X$, et al. Pre-ischemic exercise alleviates oxidative damage following ischemic stroke in rats. Exp Ther Med 2014;8(4):1325-9.

[9] Lucas SJE, Tzeng YC, Galvin SD, et al. Influence of changes in blood pressure on cerebral perfusion and oxygenation. Hypertension 2010;55(3):698-705.

[10] Vanroy C, Feys H, Swinnen A, et al. Effectiveness of active cycling in subacute stroke rehabilitation: a randomized controlled trial. Archives of Physical Medicine and Rehabilitation 2017;98(8):1576-85.e5.

[11] William JP, Colin PD, José B, et al. 2015 American Heart Association/American Stroke Association Focused Update of the 2013 Guidelines for the Early Management of Patients With Acute Ischemic Stroke Regarding Endovascular Treatment: A Guideline for Healthcare Professionals From the American Heart
Association/American Stroke Association. Stroke 2015;46(10):3020-35.

[12] Sundseth A, Thommessen B, Rønning OM. Outcome after mobilization within 24 hours of acute stroke: a randomized controlled trial. Stroke 2012;43(9):2389-94.

[13] Matsuda F, Sakakima H, Yoshida Y. The effects of early exercise on brain damage and recovery after focal cerebral infarction in rats. Acta Physiologica (Oxford, England) 2011;201(2):275-87.

[14] Park SW, Lee KJ, Shin DC, et al. The effect of underwater gaita training on balance ability of stroke patients. J Phys Ther Sci 2014;26(6):899-903.

[15] Bonita R, Beaglehole R. Recovery of motor function after stroke. Stroke 1988;19(12):1497-500.

[16] Gibala MJ, Little JP, MacDonald MJ, et al. et al. Physiological adaptations to low-volume, high-intensity interval training in health and disease. J Physiol 2012;590(5):1077-84.

[17] Guyton AC, Hall JE. Textbook of Medical Physiology. 10 ${ }^{\text {th }}$ edn. Saunders 2000.

[18] Jahani M, Modaresi MH, Mansouri K. Hypoxia inducible factor: It's role in angiogenesis and tumor. Journal of Tehran Univ Med J 2016;73(11):757-66.

[19] Austin MW, Ploughman M, Glynn L, et al. Aerobic exercise effects on neuroprotection and brain repair following stroke: a systematic review and perspective. Neuroscience Research 2014;87:8-15.

[20] Wang Y, Li M, Dong F, et al. Physical exercise-induced protection on ischemic cardiovascular and cerebrovascular diseases. Review article. Int J Clin Exp Med 2015;8(11):19859-66.

[21] Ding YH, Luan XD, Li J, et al. Exercise-induced overexpression of angiogenic factors and reduction of ischemia/reperfusion injury in stroke. Current Neurovascular Research 2004;1(5):411-20.

[22] Guo M, Lin V, Davis W, et al. Preischemic induction of TNF$\alpha$ by physical exercise reduces blood-brain barrier dysfunction in stroke. J Cereb Blood Flow Metab 2008;28(8):1422-30.

[23] Zhang P, Zhang Q, Pu H, et al. Very early-initiated physical rehabilitation protects against ischemic brain injury. Front Biosci (Elite Ed) 2012;4:2476-89.

[24] Nourshahi M, Hedayati M, Nemati J, et al. Effect of 8 weeks endurance training on serum vascular endothelial growth factor and endostatin in Wistar rats. Koomesh 2012;13(4):474-9.

[25] Cherezov V, Rosenbaum DM, Hanson MA, et al. Highresolution crystal structure of an engineered human beta2-adrenergic g protein-coupled receptor. Science 2007;318(5854):1258-65

[26] Vogel J, Kruse C, Zhang M, et al. Nox4 supports proper capillary growth in exercise and retina neovascularization. The Journal of Physiology 2015;593(9):2145-54.

[27] Taheri CH, Nourshahi M, Ranjbar K. The response of vascular endothelial growth factor to exhausted sub maximal exercise and its correlation with $\mathrm{VO}_{2}$ max. Sport Biosciences (Harakat) 2011;2(7):59-75.

[28] Naderi S, Mohammadi AR, Zadeh AS, et al. The effect of exercise preconditioning on $\mathrm{s}$ troke outcome in an experimental mice model. Shefaye Khatam 2015;3(3):4553. 
[29] Gunes H, Kandis H, Saritas A, et al. The relationship between ischemic stroke and weather conditions in
Duzce, Turkey. World Journal of Emergency Medicine 2015;6(3):207-11. 